\title{
From Current Science to School - the Facets of Green Chemistry on the Example of Ionic Liquids
}

\author{
Daniel Rauber ${ }^{1,2}$, Frederik Philippi ${ }^{1}$, Johann Seibert ${ }^{1}$, Johannes Huwer ${ }^{1,3}$, Harald Natter ${ }^{1}$, Rolf Hempelmann ${ }^{1,2, *}$ \\ ${ }^{1}$ Department of Physical Chemistry and Didactics of Chemistry, Saarland University, 66123 Saarbrücken, Germany \\ ${ }^{2}$ Transfercenter Sustainable Electrochemistry, Saarland University and Kist Europe, 66125 Saarbrücken, Germany \\ ${ }^{3}$ Department of Chemistry and its Didactics, School of Education Weingarten, 88250 Weingarten, Germany \\ *Corresponding author: r.hempelmann@mx.uni-saarland.de
}

Received January 15, 2019; Revised February 26, 2019; Accepted April 03, 2019

\begin{abstract}
Ionic liquids (ILs) are a widely investigated topic in various technical disciplines at the present time. A main focus of the research on ILs lies on applications in the field of a more sustainable, 'greener' chemistry that benefits from their unique property combination. This allows for the improvement of existing technologies and the use in novel, innovative processes. Especially the synergistic combination with other concepts of green chemistry is expected to lead to promising application of ILs. Regardless of their presence in current scientific research, they are covered rarely in the didactics of chemistry, although they represent an ideal subject to incorporate fundamental relationships or chemical concepts into teaching. They can also be included in various demonstration or hands-on experiments. Therefore, the topic of ILs is very promising for the transfer of knowledge from chemical research and industry into didactical experiments especially in the greater context of green chemistry. In this contribution, we present some simple experiments that utilize a multifunctional IL as recyclable catalyst in a biphasic homogenous catalysis. The experiments demonstrate the ecological and economic benefits offered by ILs in an intelligent process design compared to the conventional protocols for esterification. It is shown that exploiting the potential offered by the tunability of functional ILs as high-performance chemicals rather than 'simple solvents' offers many possibilities for a more sustainable chemical production.
\end{abstract}

Keywords: ionic liquids, green chemistry, sustainable chemistry, knowledge transfer, esterification, biphasic homogenous catalysis, recycling

Cite This Article: Daniel Rauber, Frederik Philippi, Johann Seibert, Johannes Huwer, Harald Natter, and Rolf Hempelmann, "From Current Science to School - the Facets of Green Chemistry on the Example of Ionic Liquids." World Journal of Chemical Education, vol. 7, no. 2 (2019): 153-165. doi: 10.12691/wjce-7-2-15.

\section{Introduction}

Ionic liquids (ILs) are a novel class of tunable liquid materials that show an advanced and unique property combination. Per historical and arbitrary definition, an IL is a salt melt with a melting point below $100{ }^{\circ} \mathrm{C}$. For practical implementations, the subclass of room-temperature ionic liquids (RTILs), containing ILs with melting points below $25^{\circ} \mathrm{C}$, is of highest interest.

The composition solely of ions results in a unique combination of characteristics that is significantly different from those found for molecular solvents. These properties include intrinsic conductivity and often a wide electrochemical window that makes the ILs promising candidates as electrolytes for various electrochemical applications. These cover for instance super capacitors, batteries and dye-sensitized solar cells (DSSCs) that are important devices for the storage and conversion of energy in the context of the expanding renewable energy usage $[1,2]$. Furthermore, ILs have a negligible vapor pressure what hinders their evaporation into the environment and the exposure of operators via the gas phase. In addition, ILs show usually high thermal stabilities which enables their usage at elevated temperatures and protects devices such as batteries efficiently against ignition or explosion in cases of incidents.

Maybe the most striking property of ILs is their adaptability towards desired properties or functions by the choice of cation and anion, the attachment of functional groups etc. Because of this, ILs are sometimes called 'designer solvents' [3] or 'task-specific fluids' [4]. Their phase behavior, for instance, can be varied in a wide range from highly hydrophilic to highly lipophilic which is often exploited for the recycling of ILs. Due to their combination of features, ILs are sometimes regarded as the link between conventional, molecular liquids and high temperature molten salts.

In their early days (and sometimes even today) ILs were generally portrayed as 'green solvents', especially when compared to conventional molecular solvents, belonging to the class of volatile organic compounds (VOCs). This attribution was based on their beneficial properties, such as intrinsic safety, non-volatility and recyclability if used as solvents for a particular reaction. Although these characteristics are important features for 
neoteric, sustainable solvents there is now clear evidence that ILs are often not a green substitute for VOCs if the overall environmental impact is considered [5]. This will later be discussed in more detail. However, the carefully considered usage of the low temperature molten salts can undoubtedly have a positive influence on the sustainability of a process, device or reaction, where the IL is applied. Irrespective of these controversies, the field of ILs is still a highly promising and widely investigated field of green chemistry although not all of the initial promises on sustainability are kept [6].

Although ILs are a widespread topic with large impact on many fields of science, especially chemistry, since the discovery of air and moisture stable representatives in the early 1990s, they are nearly absent in the didactics of chemistry $[7,8]$. The underlying reasons for this may be in the requirement of a well-developed expertise in chemistry and related sciences and the still comparably high costs of ILs. However, the field of ILs offers unique possibilities for the teaching of chemistry as it combines up-to-date research with recent trends in social and technical developments all over the globe. The topicality of an issue is in general very beneficial for increasing the motivation of the learners. In combination with the area of green chemistry, there is also a quite singular chance to show sustainability and the way it is implemented in particular experiments rather than pointing out or discussing solely theoretical concepts. With focused teaching, the learners can be sensitized for sustainable development. The field of ionic liquids can also be combined with the teaching of chemical concepts, for instance the structure-property relation and the fluent transition in properties from molecular to ionic components. Realization of the approaches for greener and more sustainable chemistry is inherently required for future generations due to the problems resulting from the human impact on the environment. The negative impact is multifaceted and ranges from the local problems of contaminations by persistent, toxic chemicals (for instance from mining or industrial chemical production) to global issues (such as the anthropogenic climate change, ozone depletion and pollution of maritime habitats).

In this contribution, we will give a short introduction on the class of ionic liquids and discuss their usages in the context of green and sustainable chemistry critically. The combination of ILs and green chemistry are ideal for the transfer of scientific knowledge into chemistry didactics as they combine recent scientific research and the resulting controversies with transformations in society and industry. By this combination it is also shown how science and social transformations are interrelated and how scientific research tries to face problems in the framework of global developments. It will furthermore be shown how ILs can contribute to improvements in very diverse technologies that are all associated with the context of sustainable development but in many different ways. The discussed concepts will then be demonstrated by simple didactical experiments using a multifunctional IL as a less energy and chemical intensive alternative to conventional chemistry. It is shown that combining ILs with other green chemistry concepts gives synergistic effects that allow less energy consuming separations, solvent reduction and easy regeneration of the IL catalyst in a closed-loop process.

\section{Ionic Liquids and the Green Chemistry Principles}

The need for green and sustainable chemistry is addressed in many sustainability goals of the UN development plan 'Transforming our world: the 2030 Agenda for Sustainable Development' from 2015 [9]. The concepts of sustainability contain the three interwoven factors of ecologic, economic and social sustainability. For chemistry this means reduced cost due to more efficient processing and minimized utilization of energy and chemicals. This demands furthermore the increased exploitation of renewable feedstocks and chemicals that are inherently safe to operators and environment. In combination with optimized recycling strategies waste and release of chemicals into the environment can be prevented. To get an insight into the influence of ionic liquids on novel, more sustainable technologies, the knowledge about their fundamental properties, especially in comparison to conventional solvents, is of vital importance. Some of the most important molecular structures of IL constituting cations and anions and the resulting properties of ILs are summarized in Figure 1.

\section{IL cations IL anions

Imidazolium-
Itonic

Figure 1. Molecular structures of some important cations and anions used for the formation of ionic liquids and notable characteristics in the context of green and sustainable chemistry

Solvents and their reduction are a field of growing interest in the chemical industry and science. They are used for homogenous reaction conditions, heat control and to drive an organic reaction towards a desired outcome [10]. Compared to the actual reagents, the quantities of solvents used are usually much higher. Generally, mostly the impact on the environment induced by solvents is therefore larger than the impact resulting from the actual chemical reaction. Additional solvent amounts, apart from 
those used for the chemical transformations, originate from extraction and purification processes where large amounts of solvents are commonly necessary. The annual production of organic solvents was recently estimated to be almost 20 million metric tons [11]. As the vast amounts of solvents are VOCs, they have an immense impact on pollution and energy consumption. VOCs therefore directly influence the quality of life (by contamination of air, water and soil) and have an impact on climate change (for instance by generation of solvents from fossil fuel stocks or energy usage in their removal etc.). The practice of solvent usage in the past and today with excessive consumption of non-renewable, toxic, volatile and inflammable solvents is an exceptional example for a chemistry that isn't sustainable at all. However, this is quite useful from a chemistry didactics point of view to demonstrate the need for more sustainable solvent approaches and possible solutions offered by the recent scientific research. This points out that the chemical industry is accountable for environmental and health related problems (which is well recognized by the public) on the one hand, but on the other the chemical industry underpins improvements and solutions to recent problems (this being often unrecognized by the public) [12]. The search for more environmentally friendly, neoteric solvents is motivated by the reduction of the excessive negative impact of conventional solvents that contribute significantly to the influence of chemistry overall. Therefore, the search for sustainable solvent solutions seems to be the most active area of green chemistry research today [13]. Among the classes of neoteric solvents, the family of ILs is by far the most versatile with many additional features that could be used in a multifunctional way. As already mentioned, the conventional solvents used in labs and industry are often volatile and inflammable, toxic, hazardous to the environment, depend on fossil fuel stocks and contribute to the greenhouse effect. The aim of using neoteric solvents is to eliminate these effects to the highest degree possible to provide safe, biodegradable and environmentally harmless solvents from renewable resources with low energy consumption for their production. The reduction of solvent consumption and their substitution by less critical liquids (for instance the replacement of chlorinated solvents by ethanol) or even by water are general strategies to fulfill official requirements and to reduce the costs for solvent waste disposal. However, for the majority of reactions, this is often chemically restricted or economically not justifiable. Recycling of neoteric solvents is often subject to economic criteria, in contrast to the green imperative for the case of conventional solvents. This demands a multidisciplinary approach for the field of green and sustainable chemistry, that is still in the state of development, as there are the already mentioned inherent interconnections between factors of sustainability [14].

Many of the criteria for more sustainable solvents are generally fulfilled by ILs. Therefore, they were portrayed as overall 'green solvents' [15] in the earlier years of research (and even sometimes today). When considered as solvents for a particular reaction, ionic liquids always offer the advantage of non-volatility; thus evaporation in the environment and exposure of operators can be avoided. Furthermore, the negligible vapor pressure and thermal stability prevents accidents by inflammation or explosions as well as thermal runaways (for instance from exothermic reactions or in the case of incidents when used in technical devices). Due their high stability and tunable phase behavior, they are also predestined for recycling processes to reduce the overall solvent consumption. While the molecular organic solvents are limited to only a few hundred representatives, the number of potential ILs was estimated to be as high a $10^{12}[16]$. The immense number of possible ILs and chemical functionalization offers the design of ILs with tailored characteristics for particular tasks to reduce the expense of chemicals and energy. Because of the adjustability and dual organic and ionic nature, the ILs are also powerful solvents for a wide range of organic and inorganic compounds that are hardly soluble in conventional solvents. This is highly attractive for the separation and processing of biological macromolecules, for instance lignin and cellulose that stem from renewable resources [1]. Also, the realization of multifunctional liquids with 'beyond solvent' characteristics, for instance catalytic activity, magnetic properties or a role as chiral induction agent, are well established in the field of ILs. This allows several innovative strategies for further reduction of resource consumption for instance as combined solvent and catalyst. In particular reactions, ILs often fulfill most of the twelve green chemistry principles as formulated by Anastas and Warner [17]. These principles serve as a guideline to achieve a more sustainable chemistry and can be summed up in the following way [13].

Waste prevention: Any waste of energy or chemicals should be avoided to the highest amount possible since waste removal demands further resources.

Maximize atom economy: Chemical reactions should be designed in a way that the maximum amount of initial materials can be found in the final product.

Reduce hazards in synthesis: If possible, the synthetic strategies should choose substances with low toxicity and lower potential hazards.

Design of safer chemical products: New chemicals with retained efficiency and function but reduced toxicity should be designed.

Safer solvents and auxiliaries: The application of auxiliary substances (for instance solvents and separation agents that are not participating in the actual reaction) should be avoided wherever practical as this demands additional chemicals and energy. If they are unavoidable they should be as safe as possible.

Energy efficient design: Chemical processes should be optimized to consume less energy since the conversion of energy has a large environmental impact. Therefore, reactions at ambient conditions are to be preferred.

Usage of renewable feedstocks: Materials should be derived from renewable feedstocks rather than depleting resources (such as mineral oil and metals).

Reduction of derivatives: Derivatization steps should be avoided if practicable since they demand additional synthetic steps and usually large amounts of chemicals.

Catalytic reactions: Selective catalysts should be preferred over stoichiometric reagents.

Degradability of products: Chemical products should be designed to break down into harmless degradation products to avoid persistence in the environment. 
Table 1. Fulfillment of the green chemistry principles by utilization of ILs in various functions

\begin{tabular}{|c|c|c|}
\hline \multicolumn{2}{|c|}{ Green Chemistry Principle } & \multirow[b]{2}{*}{$\begin{array}{l}\text { Contribution of ionic liquids } \\
\text { - No losses through evaporation/ aiming for and adjusting of properties for high recycling rates } \\
\text { - High stability often avoids additional cleaning steps (from degradation products) and enables full } \\
\text { recycling } \\
\text { - Improvement of reaction rates, selectivity and yields possible (by solvent effects, for instance via } \\
\text { stabilization/destabilization of intermediates and favored reaction pathways) } \\
\text { - Often implementation in long-term applications desired (technical devices such as batteries, } \\
\text { supercapacitors, processes with included IL recycling) }\end{array}$} \\
\hline 1 & Waste prevention & \\
\hline 2 & Atom economy & - No negative influence/ usually not used as initial material \\
\hline & Reduce hazards & $\begin{array}{l}\text { - Hazards associated with volatile compounds (inhalation, inflammability) are eliminated } \\
\text { - ILs can be designed in nontoxic way [18] }\end{array}$ \\
\hline 4 & Safer products & $\begin{array}{l}\text { - Non-volatility makes ILs much safer in transport and applications than volatile, inflammable solvents } \\
\text { - ILs often show high stabilities and thermal decomposition with much lower pressure build up in case of } \\
\text { incidents (of relevance for instance as electrolytes in energy storage applications) } \\
\text { - ILs can be combined with other materials to yield novel, safe materials (ionic liquid crystals, } \\
\text { polymerized ionic liquids, ionogels,...) [19] }\end{array}$ \\
\hline 5 & Reduced auxiliaries/ safer solvents & $\begin{array}{l}\text { - Good and adjustable dissolution behavior can be exploited to reduce solvents as well as reaction or } \\
\text { extraction steps } \\
\text { - Multifunctional IL can help in the reduction of chemicals } \\
\text { - ILs are usually much safer than molecular solvents }\end{array}$ \\
\hline 6 & Energy efficiency & $\begin{array}{l}\text { - Novel processes with increased energy efficiency possible (for instance multiphasic catalysis or reactions } \\
\text { at elevated temperature without need for pressurization of the reaction vessel) }\end{array}$ \\
\hline 7 & Usage of renewable feedstocks & $\begin{array}{l}\text { - ILs allow very promising routes for the further utilization of renewable feedstocks due to their strong } \\
\text { solvation ability for biological macromolecules such as lignin and cellulose } \\
\text { - ILs themselves can be made from renewable feedstocks [20] }\end{array}$ \\
\hline 8 & Reduction of derivatives & - Usually no negative impact as ILs are not used for derivatization \\
\hline & Catalytic reactions & $\begin{array}{l}\text { - Some ILs were proven to catalyze certain reactions (mainly Lewis or Brønsted acidic/ basic ILs) } \\
\text { - Some ILs can support catalysis (activation of catalyst, stabilizing catalytic active species) }\end{array}$ \\
\hline & Degradability of products & $\begin{array}{l}\text { - ILs can be designed to be biodegradable [21] } \\
\text { - Some ILs also occur in nature [22] }\end{array}$ \\
\hline & Real-time analysis & - No influence (real-time analysis can also be applied to reactions with ILs) \\
\hline & Safer chemistry & $\begin{array}{l}\text { - ILs make chemical processing safer compared to volatile, inflammable solvents } \\
\text { - No pressure build up at higher temperatures } \\
\text { - High thermal stability prevents accidents in case of operator errors or technical incidents }\end{array}$ \\
\hline
\end{tabular}

Real-time analysis for pollution prevention: Analytical techniques for real-time monitoring of chemical processes should be used to avoid the formation of hazardous substances and side products.

Inherently safer chemistry to prevent accidents: Substances and their form should be chosen to minimize the potential for incidents, releases, fires and explosions.

The beneficial influence on reactions of ILs in the context of the green chemistry principles are given in Table 1 .

The advantages of ILs to meet the criteria of green chemistry as safe, recyclable solvents compared to organic solvents for most reactions are obvious. Although the price of ILs is at present much higher than the one of molecular solvents, this disadvantage might be overcome in the future by optimized synthetic routes, by consequent recycling and by extended usage of ILs. However, to judge the entire sustainability of ionic liquids usage, there are many more factors that must be considered. In the overall assessment, leaving economic considerations aside, there are other factors that prevent the portrayal of IL to be totally 'green' which has been subject to ongoing controversies in the scientific community [23,24]. For example, it has to be taken into account that, although the elimination of conventional solvents can commonly be realized with ILs, this is not the case for the whole production and usage cycle. As ILs are more complex than molecular solvents, they demand much more steps in their synthesis. This is accompanied by increased consumption of energy and chemicals as well as a production of waste for the production of ILs. For many ILs used today, the life cycle assessment (LCA) has revealed that substituting organic solvents with ILs would merely shift the environmental impact to the production stage [25]. In addition, many of the chemicals used for the synthesis of ILs such as alkylation agents are usually hazardous. And are obtained from non-renewable feedstocks. Diminishing or even eliminating these problems was demonstrated by the usage of ILs from renewable feedstocks [26] and the application of solvent-free methods in the IL's synthesis [27].

The high chemical stability and low volatility of ILs are desirable characteristics for chemical processing but are a matter of concern from the point of (bio-)degradability. Biodegradation studies often investigate the ILs behavior under particular conditions and are unable to cover this highly complex area [28]. Also, the majority of ILs is water-soluble so the impact on the aquatic milieu and soil as well as on sorption/desorption cannot be neglected [29]. Many of the ILs that are commercially available today were found to have a low biodegradability and may therefore be stable and persistent environmental pollutants. Furthermore, the toxicity of ionic liquids on different forms of life is rarely investigated depends on the particular organism. As a result, any developments for new, more sustainable ILs focus on the environmental fate and toxicity of ionic liquids in order to design ILs that are biodegradable and possess low toxicity [30]. 
Overall, the usage of ILs as 'simple solvents' to substitute molecular liquids is more critically addressed today since the promised economic benefits are often not or only partially fulfilled by the utilization of ILs. The usage of ILs as widespread substitutes for organic solvents therefore seem to be a very unrealistic scenario [31]. However, low cost ILs from renewable resources with low toxicity and biodegradability may offer a practical alternative to conventional solvents in some applications, but to reach this goal there are still lots of efforts to be made. Much more auspicious is the use of ILs as high-performance chemicals for particular, carefully thought out applications that exploit their special property combination. Especially the use of ILs with multiple, tailored functions are anticipated to find their implementations in the chemical industry to conserve chemicals and energy. It should be noted that even if ILs are not a greener alternative for solvents or processes, they may nevertheless have a big influence on other technologies necessary for sustainable development [2]. For instance, many efforts towards developing safer batteries or supercapacitors with improved characteristics (such as capacity and lifetime) focus on ILs as electrolytes. In this way, ILs may contribute as high-performance chemicals significantly to the further distribution of electrically driven vehicles. This goes hand in hand with the reduction of non-renewable energy usage and air pollution.

Other very promising and more sustainable approaches utilizing ILs are in the field of pretreatment or processing of biomass, mainly lignin and cellulose. These pathways for the expanded usage of renewable resources are opened by the exceptional dissolution abilities of ILs for biopolymers. Most of these biopolymers are insoluble in water and organic solvents. The need for novel processes and the innovative possibilities offered by ionic liquids for a more sustainable chemistry in the context of renewable feedstock exploitation will be explained for two examples: the industrial cellulose processing and the lignin usage. In the case of cellulose, the established, market-dominating industrial method (the so called viscose process) applies the highly toxic and inflammable carbon disulfide $\mathrm{CS}_{2}$ to convert cellulose into cellulose xanthate which is soluble in alkaline aqueous solutions. The cellulose is then regenerated by spinning the xanthate solutions in aqueous sulfuric acid solutions. This large-scale process to produce viscose rayon or cellophane consumes huge amounts of chemicals and energy and produces a lot of waste. Although the viscose process supports the usage of renewable resources, it is therefore not a sustainable process in its entirety as it violates many of the green chemistry principles. Therefore, the searches for alternative processing methods that fulfill the green chemistry principles to a higher degree are strongly demanded. ILs were identified as ideal candidates for this purpose. They are much safer, can be recycled easily by spinning into water baths and can dissolve large amounts of cellulose without the need for additional derivatization agents. This offers not only novel, more sustainable cellulose processing methods that are currently already advanced to pilot scales but also innovative biodegradable materials with additional functionalities [32]. Further substitution of mineral-oil based products, mainly polymers, with the biocompatible and biodegradable cellulose products would also have a positive influence by reducing the plastic pollution in maritime habitats. Similar problems as faced in the case of cellulose hold true for the exploitation of lignin. Lignin is a main constituent of woody plants and forms composites with cellulose in their cell walls (the lignocellulose that is mainly the dry mass of wood). In the highly energy intensive methods for the isolation of cellulose from biomass (such as sulfite, kraft and organosolv process), the lignin is obtained as a side product in megaton scale. As there is currently a lack of appropriate uses it is mainly burned as low-grade fuel to satisfy the energy demand of the cellulose isolation processes. A reason for the absence of lignin utilization is its insolubility in many solvents. With ILs as powerful, inert and recoverable solvents for lignin, novel strategies for the usage of the aromatic biopolymer are discussed in the scientific community [33]. These approaches utilize either cracking into useable chemicals by different methods, to have a fossil-fuel independent, large-scale source for aromatic molecules or the incorporation into biodegradable polymers. The design of ILs for the selective dissolution of one biopolymeric constituent of lignocellulose (usually the aims undertaken are for the lignin-component) could also offer a less energy intensive alternative to the biomass processing combined with the generation of lignin solutions for further conversion. We have already developed demonstration and hands-on experiments covering ILs in energy-storage and conversion devices (supercaps, Li-ion-batteries and DSSCs) where we show their beneficial influence in detail [1]. In addition, we have also developed a series of experiments covering the usage of ILs in the treatment and processing of renewable materials into products (such as cellulose fibers, cracking of lignin and lignin derived polymers) [1,34].

As discussed, the facets of green and sustainable chemistry are very versatile and ILs and their various usages are perfect subjects to show the ambiguities and difficulties in their implementation into more sustainable techniques. However, a more detailed scientific background for the didactic professionals is always demanded in this context to recognize all these aspects (the same applies to the research community that has not always a critical view on the subject). We have already shown the transfer of scientific and industrial research aims into didactical experiments [1]. The field combines state-of-the-art developments and can also be combined with other green chemistry approaches such as multiphasic liquid-liquid systems to obtain synergistic benefits. This combined approach achieves an overall higher degree of sustainability than the single separated approaches, as will be demonstrated in the experiments below. The many diverse aspects covered by this field offer a flexible handling with individual foci depending on the didactic aims and contexts that should be covered.

\section{Practical Industrial Implementations of Green Chemistry Approaches}

As background for the transfer of current scientific and industrial methods into didactical experiments, we want to 
highlight two large-scale processes that already implement many green chemistry principles. These are the Ruhrchemie/Rhône-Poulenc (RCH/RP) process for hydroformylation and the BASIL ${ }^{\mathrm{TM}}$ (Biphasic Acid Scavenging utilizing Ionic Liquids) process of the BASF SE. Both processes use multiphasic reaction conditions to reduce energy and chemical input. They are the successful model techniques for the following didactic experiments so their underlying principles and advantages over conventional reaction control are essential for understanding.

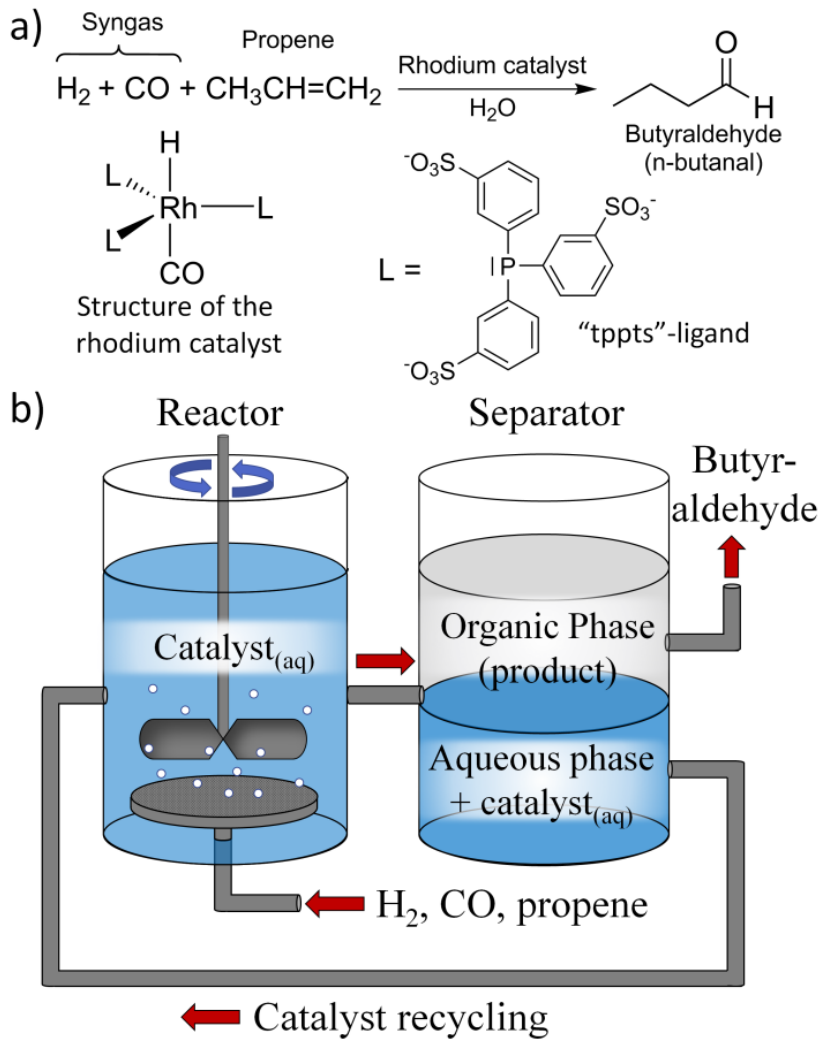

Figure 2. Scheme of the Ruhrchemie/ Rhône-Poulenc oxo process. a) reaction scheme and structure of the rhodium catalyst. b) Formation of biphasic liquid mixture that is split in a separator to obtain the organic product phase. The catalyst remains dissolved in water and is recycled by the aqueous phas

The RCH/RP process is perhaps the most widely known example for homogenous multiphasic catalysis. It uses a highly hydrophilic rhodium catalyst for the hydroformylation of propylene to $n$-butanal with the help of syngas ( $\mathrm{a}$ one to one mixture of carbon monoxide $\mathrm{CO}$ and hydrogen $\mathrm{H}_{2}$ ). The structure of the used catalyst and the principle of the $\mathrm{RCH} / \mathrm{RP}$ process are sketched in Figure 2. Catalytic reactions can be divided into two big subclasses of homogenous and heterogeneous catalysis, in dependence of the phase where catalyst and reactants are located. In homogenous catalytic reaction both catalyst and reactants are in the same (liquid) phase. This allows low catalyst concentrations, mild reaction conditions, and often easy adjustability of the clearly defined catalyst. The drawback of this reaction type is the often very difficult recovery of the catalyst that causes high running costs through catalyst loss and catalyst renewal. In contrast to homogeneous catalysis, the active species of heterogeneous catalytic reactions are immobilized in a second phase (usually the surface atom of a solid or on a solid support) that makes catalyst recovery facile, thus reduces the expenses for catalyst renewal to a minimum. In contrast, the heterogeneous catalysis is very hard to modify and often demands harsh reaction conditions (high temperature and pressure) that lead to high costs for the conduction of the process. An approach to combine the advantages of both catalytic reactions, to reduce chemical and energetic demands, is the use of liquid multiphasic systems. The multiphase catalysis thus allows low catalyst concentrations, high activity and selectivity as well as catalyst recovery by immobilization of the catalyst in a second liquid phase. The hydrophilic modification of the catalyst, which is dissolved in water, is achieved by sulfonate-groups. The organic product formed during the reaction is not miscible with water, thus forms a second phase. Therefore, it can be isolated in an energy efficient way in a separator. The hydrophilic catalyst is immiscible in the organic phase and thus remains in the aqueous phase that is returned to the reactor. The $\mathrm{RCH} / \mathrm{RP}$ process has an annual production capacity of several hundred thousand tons with losses of rhodium catalyst in the partper-billion range $[35,36]$. The low loss of the expensive catalyst is exceptionally remarkable since such efficient recovery is usually only possible with heterogeneous catalysts. In addition, the catalyst also allows a high ratio of $n$ - to iso-butyraldehyde (96/4) as a result of the triphenylphosphine ligands with high sterical demand. This demonstrates the advantage of catalyst tunability in the homogenous catalytic reaction. As there is an immense number of ILs with phase behavior adjustable towards water and organic solvents, these neoteric solvents are very promising fluids for the further developments of multiphasic reactions with homogeneous reaction conditions and facile catalyst recovery. The ILs can also be used as alternative to water when a highly polar phase that is compatible with the reagents used is needed. A successful development of such a biphasic system including ILs was demonstrated by the BASIL ${ }^{\mathrm{TM}}$ process.

The BASIL ${ }^{\mathrm{TM}}$ process of the BASF also uses a multiphasic reaction approach for the optimization of an industrial process [37,38]. It is used for the production of intermediates for photoinitiators based on alkoxyphenylphosphines. The process is sketched in Figure 3. The hydrogen chloride formed in the reaction of chlorophenylphosphine with an alcohol is thereby quenched with 1-methylimidazole. This scavenging of $\mathrm{HCl}$ is necessary to avoid unwanted side reactions of the product. Due to the incompatibility of the products with water (hydrolysis), an aqueous extraction is not possible in this case. Therefore, the reaction was initially carried out with equimolar amounts of triethylamine resulting in a thick slurry that was hard to stir. Furthermore, the slurry was found to have unfavorable characteristics for heat transfer and the filtration needed to remove the ammonium salts. To avoid the formation of this slurry the hydrochloride is quenched in the BASIL ${ }^{\text {TM }}$ process with 1-methylimidazole to obtain the ionic liquid 1-methylimidazolium chloride (melting point of this IL is $75^{\circ} \mathrm{C}$ ), which separates as a second liquid phase (under the reactions conditions of $80{ }^{\circ} \mathrm{C}$ ) because of its higher density and polarity. As in the $\mathrm{RCH} / \mathrm{RP}$ process, the separation of the product phase and the recovery of the expensive starting materials are facile. While the solvent- 
free product forms the nonpolar upper phase, the polarionic IL forms the lower phase. After separation of the two phases, the initial 1-methylimidazol can be regenerated by neutralization of the IL with sodium hydroxide to recover the expensive chemical. The way of utilizing ILs has multifold benefits for the BASIL ${ }^{\text {TM }}$ process. First of all the 1-methylimidazole acts as nucleophilic catalyst and its utilization increased the productivity of the process by a factor of $8 \times 10^{4}$ to $690.000 \mathrm{~kg} \mathrm{~m}^{-3} \mathrm{~h}^{-1}$ [39]. This allowed the change of the reactor from a batch vessel to a small jet reactor with continuous operation. The process has a capacity of more than 1000 annual tons. On laboratory scale, the process for acid scavenging was also tested several other reactions. This novel acid scavenging process satisfies many of the green chemistry criteria such as solvent elimination, reduced expense of chemicals and energy, reduction of waste, use of catalysts and improved safety. It shows the possibilities for multifunctional use in reactions offered by the very diverse solvent class of ionic liquids for a more sustainable chemistry. In the following chapter, we will show how these current scientific and industrial technologies can be scaled down to didactic experiments that show the advantages of ILs and multiphasic reactions for green chemistry.

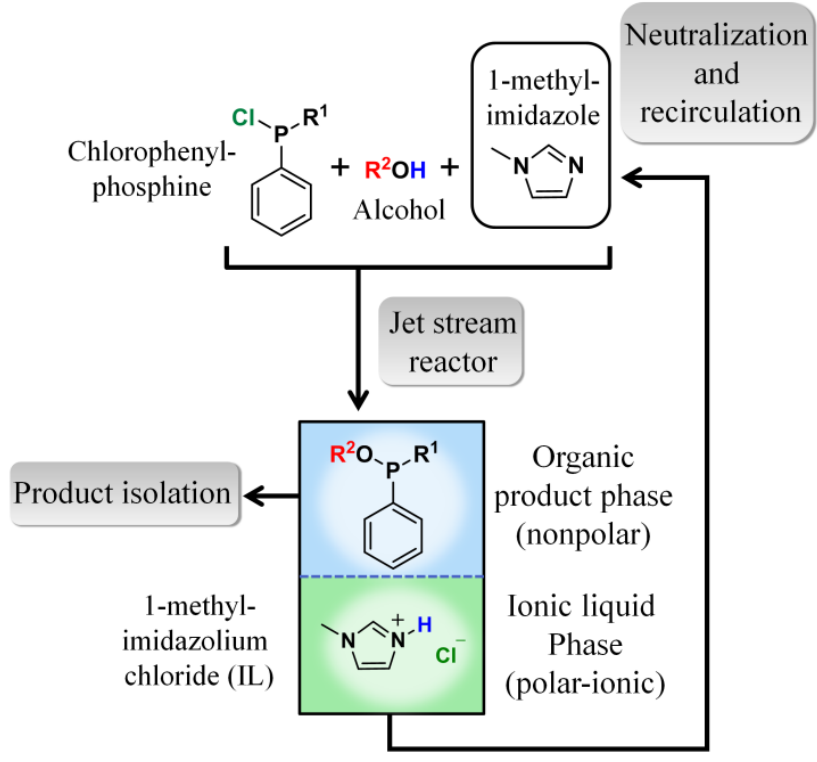

Figure 3. Scheme of the BASIL ${ }^{\mathrm{TM}}$ process. The chlorophenylphosphine reacts with the alcohol in a jet stream reactor to the alkoxyphenylphosphine. The hydrogen chloride generated in the reaction is scavenged with 1-methylimidazole forming the IL 1-methylimidazolium chloride, that is in liquid state under the applied reaction conditions. Due to the different polarity and density, a liquid biphasic system is formed that can be easily worked up. The upper phase contains thereby the solvent-free product while the ionic liquid is localized in the lower phase. The initial 1-methylimidazole is regenerated by neutralization with sodium hydroxide for reuse in further production cycles

\section{Experiments Utilizing Ionic Liquids for Multiphasic Reactions}

The following experiments are intended to show an alternative reaction processing using a multifunctional IL for Brønsted acid catalyzed esterification. The simple experiments can be performed either by lecturers as demonstration or by the learners themselves as hands-on learning. The introduced strategies are similar to the industrial realizations for more sustainable chemical processing discussed above. They can be performed either as student experiments or in the introductory courses in universities. The focus is thereby on more sustainable processing and safer solvents to reduce risks and waste while at the same time chemicals and energy consumptions are reduced. This is achieved by advanced and intelligent process design and the utilization of the many possibilities offered by ILs as a novel, innovative material class. The chapter starts with the introduction of the underlying principles followed by the description of the actual experiments and the discussion of the processing in the context of green chemistry.

\subsection{Brønsted Acid Catalyzed Esterification}

The esterification of carboxylic acids and alcohols is one of the most important and common catalytic transformations in organic chemistry. The scheme of the esterification is shown in Figure 4. The reaction demands a strong acid, such as the industrially applied sulfuric acid, as catalyst [40].

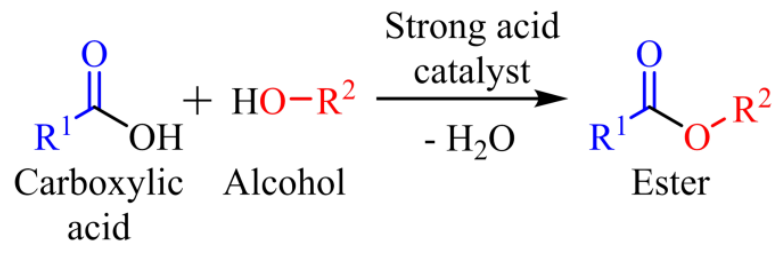

Figure 4. Scheme of the Brønsted acid catalyzed esterification of carboxylic acid and alcohol

Since the equilibrium of the reaction is on left side, several strategies for the shift to the products according to Le Chatelier's principle are applied. This is realized for instance by the addition of excess alcohol (5-10 equivalents as it is usually the cheaper component) or the removal of the water formed in the reaction with the help of a Dean-Stark apparatus or molecular sieves. In addition, many of the methods use chlorinated solvents such as chloroform to shift the reaction equilibrium by extraction of the product. These solvents are well known to pose hazards to the environment and to the operators' health. Other frequently used solvents like toluene are inflammable and also dangerous to human health when the volatile compound is inhaled. The acid catalyst used for the esterification is commonly neutralized as it can hardly be recovered, thus generating additional waste from the neutralization. The very strong acids usually applied for the esterification such as sulfuric or $p$-toluenesulfonic acid also favor side-reactions, for instance ether generation or Wagner-Meerwein rearrangements. The increased formation of side-products reduces the yield and demands for further purification steps. In addition, the product isolation of the conventional esterification usually demands further extractions steps that include volatile inflammable organic solvents that should be avoided when a more sustainable chemistry is envisaged. All in all, the conventional esterification has a large potential for optimization to follow the green chemistry principles. 
Most of the issues addressed above can be eliminated by the intelligent utilization of multifunctional ILs. The basic organic reaction of esterification is therefore a good example to demonstrate the use of ILs for a more sustainable chemistry. In addition, the simple didactical experiments presented below also do not demand advanced chemical knowledge as they rely on a quite simple reaction. In this case, the use of aqueous systems is not beneficial since it would shift the equilibrium to the reactant side.

\subsection{Multiphase Catalysis Using a Brønsted Acidic Ionic Liquid}

The following experiments utilize the ionic liquid 1-butyl-3-methylimizazolium hydrogen sulfate ([BMIM][HSO 4 ; CAS: 262297-13-2) for the esterification of different carboxylic acids and alcohols. Its molecular structure is shown in Figure 5. The experiments are designed for a minimal use of the IL to minimize the costs while the results remain clearly observable. The IL is air and moisture stable but absorbs water from the atmosphere (hygroscopic behavior). It should therefore be stored in a closed bottle. The hydrogen sulfate anion is the catalytically active species that acts as a strong Brønsted acid $\left(\mathrm{pK}_{\mathrm{a}}\left(\mathrm{HSO}_{4}\right) \approx 1.92\right)$ [41]. This minimizes side reactions in contrast to the conventionally applied very strong acids $\left(\mathrm{pK}_{\mathrm{a}}\left(\mathrm{H}_{2} \mathrm{SO}_{4}\right) \approx-3\right)$ [41]. Due to its ionic structure it is a polar, viscous liquid that is able to dissolve both the polar carboxylic acids and the alcohols. As the formed products are nonpolar, they are insoluble in the ionic liquid which results in the formation of a second phase during the course of the reaction [42]. This is similar to the industrial methods discussed above that utilize multiphasic systems for easy processing and energy-conserving product separations.

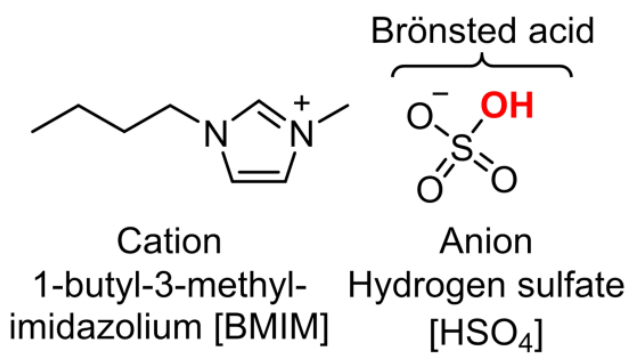

Figure 5. Molecular structure of the ionic liquid 1-butyl-3-methylimidazolium hydrogen sulfate used as catalyst for the esterification

Furthermore, this reaction processing eliminates additional solvent use and offers facile catalyst recovery. The multifold benefits for the IL use as combined catalyst and solvent for a more sustainable esterification can be summed up in the following way $[43,44,45]$ :

Use as combined solvent, catalyst and dehydrating agent (extractive esterification).

Reduction of side reactions due to the use of strong acid (hydrogen sulfate) rather than of a very strong acid (sulfuric acid).

Elimination of volatile, inflammable and hazardous solvents to increase safety
Use of efficient catalyst rather than excess reagents.

Energy efficient separation of product.

Regeneration of the catalyst/IL instead of neutralization and disposal.

\subsection{Experiments for More Sustainable Esterification with Ionic Liquids}

The following hands-on experiments are intended to show the learners a more sustainable esterification with the aid of the multifunctional ionic liquid [BMIM] [HSO 4 . The IL is commercially available from various suppliers or can be synthesized following literature protocols $[46,47,48]$. The first experiments utilize only $1 \mathrm{~mL}$ of $[\mathrm{BMIM}]\left[\mathrm{HSO}_{4}\right]$, which is already sufficient to follow the progression of the reaction that becomes observable by the formation of a second phase. Further reaction progression is also traceable by the characteristic scent of the ester products formed. This eliminates the need for additional, expensive and long lasting analytical methods (Surely, the products could also be identified by routine methods such as NMR or IR spectroscopy). The reactions do not require expensive laboratory equipment and proceed quite fast so that the experiments are also performable in a lesson. The further experiments utilize a larger amount of IL in a round bottom flask with reflux condenser and are aimed at optimizing the reaction yield. In this way, also the synthesis of the low-boiling ester propyl acetate, whose scents resembles rum aroma, is possible. In a final experiment, the utilized IL is regenerated to show the facile recycling for further uses. In the last chapter the reaction conduction using the IL is compared to the conventional reaction according to the principles of green chemistry.

\subsubsection{Experiments Performed in Test Tubes}

The experiments described in this chapter are aimed at showing the principles of the acid catalyzed esterification with an IL as combined solvent, catalyst and dehydrating agent. For this purpose, the reactions are performed with minimum amount of IL in a simple test tube. As both the educts and products have comparably high boiling points, no reflux condenser is necessary. The test tube is sealed with aluminum foil to avoid evaporation. The reactions proceed quite fast and the products can be identified by their characteristic sent. A more quantitative experiment is described in the next chapter.

Materials: Beaker as water bath; thermometer; magnetic stirrer with heating function; test tubes; aluminum foil; disposable syringes; disposable pipettes.

Chemicals: 1-butyl-3-methylimidazolium hydrogen sulfate (CAS: 262297-13-2; GHS05: corrosive); carboxylic acids and alcohols according to Table 2 (acetic acid (GHS02: flammable; GHS05: corrosive); propionic acid (GHS02: flammable; GHS05: corrosive); n-butanol (GHS02: flammable; GHS05: corrosive); n-pentanol (GHS02: flammable; GHS07: harmful); isoamyl alcohol (GHS02: flammable; GHS07: harmful); n-hexanol (GHS02: flammable; GHS07: harmful); n-octanol (GHS07: harmful). 
Table 2. Volumes of carboxylic acids and alcohols for the experiments in a test tube using $1 \mathrm{~mL}$ of ionic liquids

\begin{tabular}{|c|c|c|c|c|}
\hline Ester to synthesize & Carboxylic acid & Volume of carboxylic acid / mL & Alcohol & Volume alcohol/ $\mathrm{mL}$ \\
\hline Butyl acetate & Acetic acid & 0.3 & n-butanol & 0.5 \\
\hline Pentyl acetate & Acetic acid & 0.3 & n-pentanol & 0.6 \\
\hline Isoamyl acetate & Acetic acid & 0.3 & isoamyl alcohol & 0.6 \\
\hline Hexyl acetate & Acetic acid & 0.3 & n-hexanol & 0.7 \\
\hline Octyl acetate & Acetic acid & 0.3 & n-octanol & 0.8 \\
\hline Butyl propionate & Propionic acid & 0.4 & n-butanol & 0.5 \\
\hline Pentyl propionate & Propionic acid & 0.4 & n-pentanol & 0.6 \\
\hline
\end{tabular}

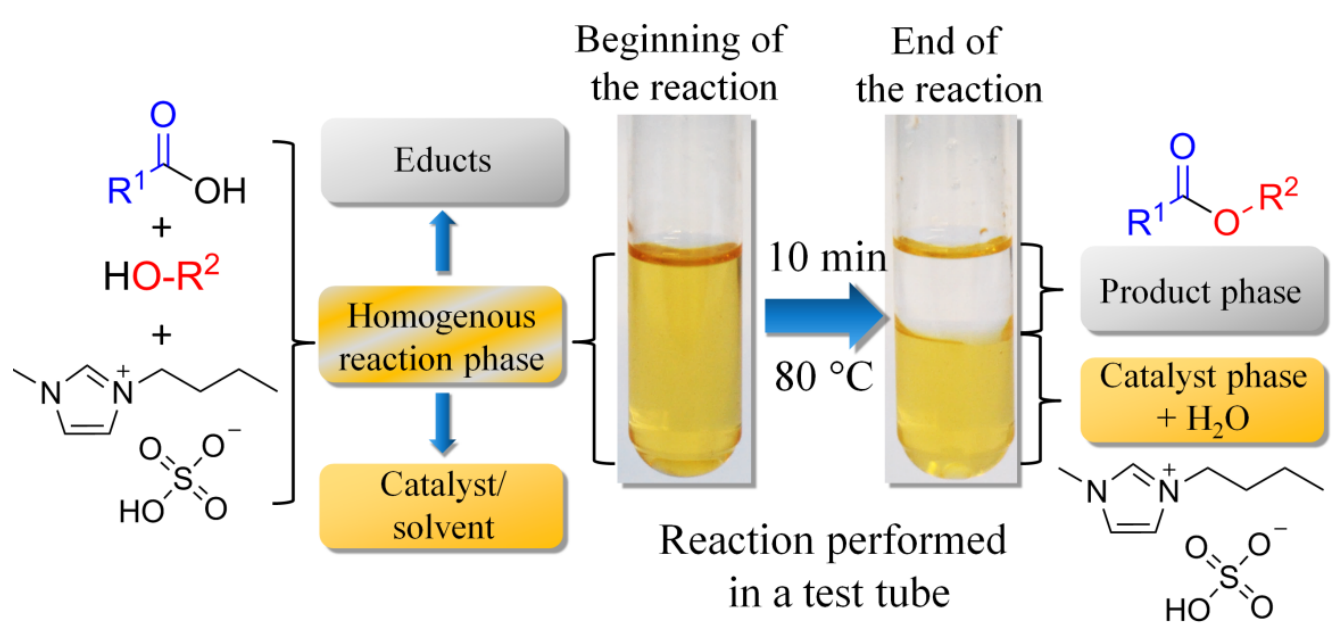

Figure 6. Reaction course of the esterification reaction with the Brønsted acidic ionic liquid [BMIM] $\left.\mathrm{HSO}_{4}\right]$ as catalyst. At the beginning of the reaction, the polar educts form a homogenous mixture with the IL. After several minutes in the water bath, a second phase of the nonpolar reaction product is formed. The separation is facilitated since the density of the product is much lower compared to the density of the IL phase

Experiment: $1 \mathrm{~mL}$ of $[\mathrm{BMIM}]\left[\mathrm{HSO}_{4}\right]$ as well as the amount of a carboxylic acids and alcohols according to the entries of Table 2 for an ester to synthesize are added to a small test tube (volumes can be measured using disposable syringes). The test tube is sealed with aluminum foil and swirled by hand for several seconds until a homogenous mixture is observed. The test-tube is placed in a water bath equipped with a thermometer and heated to $80{ }^{\circ} \mathrm{C}$ for 10-30 minutes. The progression of the reaction is monitored by visible inspection every few minutes. After cooling to ambient temperature, the upper phase is removed using the disposable pipettes. An olfactory test of the upper phase is carefully conducted. The lower phase can be gathered for subsequent recycling of the IL.

Observations: A second phase which is clear and colorless forms atop of the initial phase after a few minutes in the water bath. The volume of the lower phase is decreasing more and more during the experiment. The visible observations are shown in Figure 6. After completion of the experiment an intense scent can be perceived. These scents remind of fruits but are described to appear more artificial than the natural scent of fruits.

Explanation: The successful reaction is indicated by the formation of a second phase and the characteristic scent of the product. The separation of the two phases is facilitated by the comparably large density difference

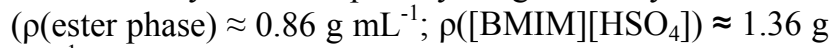
$\left.\mathrm{mL}^{-1}\right)$. The catalytic activity of the IL for the esterification is clearly visible by the product formation. The clear, monophasic reactions mixture at the beginning of the reactions shows the ability of the IL to act as solvent for a homogeneous reaction. Furthermore, the 'like-dissolves-like' principle is demonstrated in this way since the polar educts mix with the polar IL whereas the nonpolar product does not. The water formed in the reaction remains in the IL phase so that the IL acts as dehydrating agent to shift the reaction equilibrium towards the product side. The phase separation occurs by itself and allows the isolation of the product without energy or chemical intensive processes such as distillation or solvent extraction. In doing so, the esterification does not require additional solvent uses, neither for the reaction itself nor for the purification of the product. The scent of the esters reminds the learners of fruits since they are responsible, in combination with other molecules, for the natural scent of fruits. Since the natural scent of fruits is a complex mixture, the single compound product obtained here appears to be somewhat artificial.

\subsubsection{Experiments Performed in Round Button Flasks}

The following experimental part aims at a more quantitative evaluation of the reaction yield and uses a higher volume of the IL in a round bottom flask equipped with a reflux condenser. The experiments are designed for IL, carboxylic acid and alcohol in 1:1:1 ratio whereby the reaction can be scaled up according to individual requirements. Also, the preparation of the propyl acetate (scents like rum aroma) that has a quite low boiling point can be conducted with this reaction setup. The larger amount of IL used is here also helpful for the easier regeneration as shown in the next step.

Materials: Pot or crystallization dish as water bath; thermometer; magnetic stirrer with heating function; magnetic stirring bar; $25 \mathrm{~mL}$ round bottom flask (or other appropriate volumes); reflux condenser; disposable syringes; disposable pipettes, balance. 
Table 3. Volumes (V) and masses (M) of carboxylic acids (CA) and alcohols (A) for the experiments in a test tube using 5 ml of [BMIM][HSO $\left.{ }_{4}\right]$ in a round bottom flask

\begin{tabular}{|l|c|c|c|c|c|c|}
\hline Ester to synthesize & CA & M of CA $/ \mathrm{g}$ & $\mathrm{V}$ of CA $/ \mathrm{mL}$ & A & $\mathrm{M}$ of A/g & $\mathrm{V}$ of A/mL \\
\hline Butyl acetate & Acetic acid & 1.61 & 1.54 & n-butanol & 2.37 & 2.92 \\
\hline Isoamyl acetate & Acetic acid & 1.61 & 1.54 & isoamyl alcohol & 2.37 & 2.92 \\
\hline Hexyl acetate & Acetic acid & 1.61 & 1.54 & n-hexanol & 2.75 & 3.35 \\
\hline Octyl acetate & Acetic acid & 1.61 & 1.54 & n-octanol & 3.50 & 4.21 \\
\hline Butyl propionate & Propionic acid & 1.99 & 2.01 & n-butanol & 1.99 & 2.46 \\
\hline Pentyl propionate & Propionic acid & 1.99 & 2.01 & n-pentanol & 1.24 & 1.57 \\
\hline
\end{tabular}

Chemicals: 1-butyl-3-methylimidazolium hydrogen sulfate (CAS: 262297-13-2; GHS05: corrosive); carboxylic acids and alcohols according to Table 3 (acetic acid (GHS02: flammable; GHS05: corrosive); propionic acid (GHS02: flammable; GHS05: corrosive); n-butanol (GHS02: flammable; GHS05: corrosive); n-pentanol (GHS02: flammable; GHS07: harmful); isoamyl alcohol (GHS02: flammable; GHS07: harmful); n-hexanol (GHS02: flammable; GHS07: harmful); n-octanol (GHS07: harmful).

Experiment: To the $25 \mathrm{~mL}$ round bottom flask are added a weighted amount of $5.0 \mathrm{ml}$ of [BMIM] $\left[\mathrm{HSO}_{4}\right]$, a magnetic stirring bar as well as the amount of a carboxylic acids and alcohols according to the entries of Table 3 (volumes can be measured using the disposable syringes). A reflux condenser is mounted on the reaction flask. The flask is placed in a water bath with thermometer and heated to $80^{\circ} \mathrm{C}$ for 2 hours under gentle reflux. The flask is allowed to cool to ambient temperature and the upper phase is carefully separated with the disposable pipettes. The organic phase is weighted, and the yield of product is calculated $($ yield $=\mathrm{n}($ ester $) / \mathrm{n}($ alcohol $) \times 100 \%)$. The ionic liquid can be recovered in the next step and reused for further experiments.

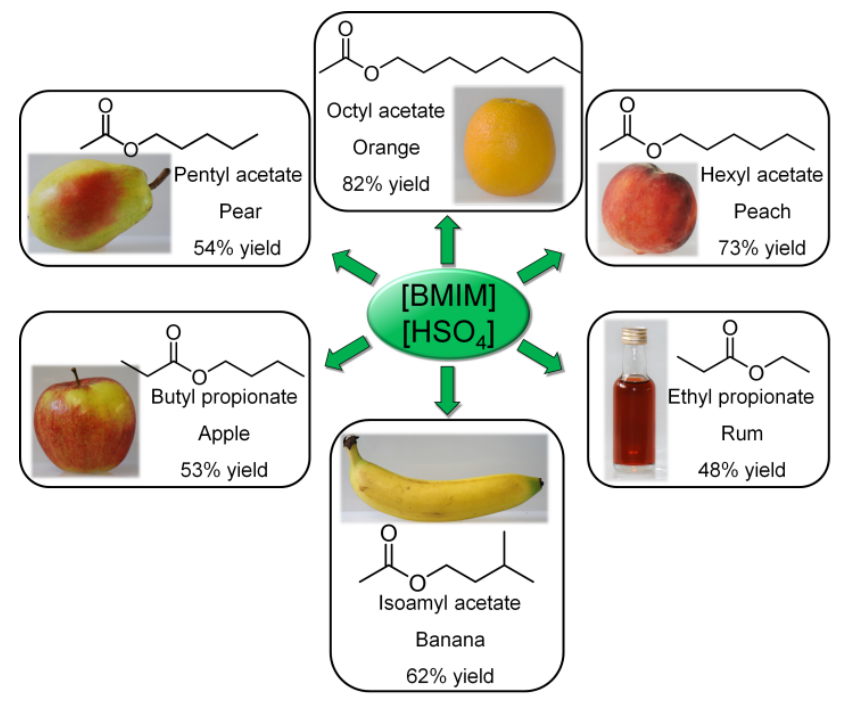

Figure 7. Molecular structures of the esters synthesized with the Brønsted acidic ionic liquid [BMIM] $\left[\mathrm{HSO}_{4}\right]$, their characteristic scents and the yields obtained after 30 minutes at $80^{\circ} \mathrm{C}$

Observations: The formation of the second phase with colorless, clear appearance above the reaction mixture is observed similar to the reaction in the test tube. The volume of the ionic liquid phase is decreasing in the course of the reaction whereas the volume of the organic phase increases. Under the applied conditions a gentle reflux is observed. The calculated reaction yields are in the range of 50 to $80 \%$. The intense characteristic scents of the esters and the reactions yields are shown in Figure 7. The lower phase shows a decreased viscosity at the end of the reaction.

Explanation: The physical principles are the same as mentioned in the previous experiment. The use of the reflux condenser reduces the evaporation to have a better estimate for the actual reaction yield. The observed yields are below the expected yields since there are losses from residues in the flask and reflux condenser. Nevertheless, the high yields from the equilibrium shift of the IL acting as dehydrating agent (extractive esterification) become apparent. Also remarkable is the very low amount of 'solvent' used when compared to conventional reactions where the solvents are often used in much higher volumes than the reactants. In addition to that, the solvent/catalyst recovery is easy to perform as shown in the next chapter. The lower viscosity of the IL phase at the end of the reaction is the result of the water formed in the reaction that weakens the coulombic interactions and, by that, the extent of the intermolecular friction.

\subsubsection{Regeneration of the Ionic Liquid Used as Catalyst}

To show the advantage of the IL for facile recycling procedures to reduce waste and chemicals, the ILs applied for the esterification is recovered in this chapter. Therefore, the IL is subjected to vacuum distillation to remove the volatile compounds, mainly the water generated in the reaction, that are dissolved in the IL. The recycling is not only attractive from ecological aspects but also from economic considerations as the IL is comparably high in prize. In this case it would be possible to generate a closed loop process with negligible losses similar to the $\mathrm{RCH} / \mathrm{RP}$ or BASIL ${ }^{\mathrm{TM}}$ process. The low amount of lost catalyst and solvent is ensured by the negligible volatility of the IL which avoids losses through the gas phase making the application of multifunctional ILs even more attractive.

Materials: Pot or crystallization dish as water bath; thermometer; magnetic stirrer with heating function; magnetic stirring bar; $25 \mathrm{~mL}$ round bottom flask (or other appropriate volumes); adapter with stopcock; washing bottle; vacuum pump (oil pump if available); vacuum tubes; balance.

Chemicals: Reaction mixture from esterification reactions (1-butyl-3-methylimidazolium hydrogen sulfate (CAS: 262297-13-2; GHS05: corrosive) with water); sodium chloride and ice (if applicable dry ice or liquid nitrogen).

Experiment: The 'consumed' IL from the esterification reactions and a magnetic stirring bar are placed in the round bottom flask. The adapter is mounted to the flask 
and connected with the washing bottle via the vacuum tubes. The washing bottle is placed in a solution of sodium chloride and ice (dry ice or liquid nitrogen if at hand) and connected to a vacuum pump. After the washing bottle is cooled down the vacuum pump is turned on. The round bottom flask is placed in a water bath then and heated to $80{ }^{\circ} \mathrm{C}$ with constant stirring. The weight of the flask is measured periodically. When no more bubble formation is observed for 30 minutes the flask is disconnected form vacuum and the amount of IL is weighted.

Observations: Upon heating the IL, bubble formation occurs and a condensate is forming in the cooled washing flask. The viscosity of the IL increases again, obtaining a fluid behavior similar to the initial IL before being used for the esterification. After approximately one hour, the weight of the flask remains constant. The measured weight is nearly the value of the IL used in the previous experiment. The IL which has been regenerated this way can be reused for further esterification (we tested this recycling without loss of activity for five times).

Explanation: The simple experiment shows the localization of the water in the ionic liquid phase rather than in the organic one (if applicable this can also be confirmed by spectroscopic methods) which is indicated by the formation of bubbles and condensate in the washing flask. The regeneration of ILs can be performed by applying vacuum since the ionic components are not volatile. In this way, the volatile molecular compounds are removed, as indicated by the bubble formation (boiling of water), leaving the purified IL in the flask. The increase in viscosity also shows the removal of water since this increases the molecular friction again. The slightly lower weight of the recovered IL can be attributed to losses from residues remaining in the equipment.

\subsubsection{Discussion of the Esterification Reactions in the Context of Green Chemistry}

After the experiments were conducted, a discussion of the observations is useful to demonstrate the different aspects of the approaches characteristic for a more sustainable chemistry. The discussion about the two esterification protocols can be freely adapted to the didactic framework and temporal conditions. The pros and contras can either be mentioned in a guided discussion, for instance according to the criteria mentioned below, or as free task to assist the learners in developing a higher sensitization for process sustainability. The comparisons of the conventionally performed esterification with the novel processing utilizing the Brønsted acidic IL are summarized in Table 4.

The ecologic and economic impacts of the IL usage are given there according to different criterions.

Both multiphasic reactions and ionic liquids as novel material class with a unique beneficial property combination have high potential for a more sustainable chemical processing and are therefore up to date research topics. Even more sustainable approaches can be established if their possibilities for safer, resource conserving and waste reducing processes are combined with other techniques in a synergistic way.

Table 4. Comparison of conventionally performed esterification and the multiphasic catalysis using the Brønsted acidic IL $[\mathrm{BMIM}]\left[\mathrm{HSO}_{4}\right]$ and the ecologic and economic impacts of the IL usage

\begin{tabular}{|c|c|c|c|}
\hline Criterion & Conventional esterification & Usage of Multifunctional IL & $\begin{array}{l}\text { Ecologic and economic impacts } \\
\text { of the IL usage }\end{array}$ \\
\hline $\begin{array}{l}\text { Production of solvent/ } \\
\text { catalyst }\end{array}$ & Comparably short production route & $\begin{array}{l}\text { More reaction steps for the synthesis of the } \\
\text { IL; not always benign reagents included }\end{array}$ & $\begin{array}{l}\text { More energy and chemicals } \\
\text { consumed in the synthesis of } \\
\text { the IL }\end{array}$ \\
\hline Solvent usage & $\begin{array}{l}\text { Often chlorinated or inflammable } \\
\text { solvents }\end{array}$ & No additional solvent needed & $\begin{array}{l}\text { Conservation of energy, } \\
\text { chemicals and reduction of } \\
\text { waste; increased safety }\end{array}$ \\
\hline $\begin{array}{l}\text { Separation and } \\
\text { purification of products }\end{array}$ & $\begin{array}{l}\text { Energy- intensive processes } \\
\text { (distillation or solvent extraction) }\end{array}$ & Simple phase separation (gravitational) & $\begin{array}{l}\text { Conservation of energy and } \\
\text { saving of time }\end{array}$ \\
\hline Catalyst recycling & $\begin{array}{l}\text { Usually not performed; neutralization } \\
\text { and disposal }\end{array}$ & $\begin{array}{l}\text { Full recovery of the catalyst without activity } \\
\text { loss; no additional chemicals needed }\end{array}$ & $\begin{array}{l}\text { Conservation of chemicals; } \\
\text { reduction of waste }\end{array}$ \\
\hline Solvent recovery & $\begin{array}{l}\text { Challenging and energy intensive; } \\
\text { usually not applied }\end{array}$ & $\begin{array}{l}\text { Full recovery of the solvent by simple } \\
\text { removal of volatile compounds }\end{array}$ & $\begin{array}{l}\text { Conservation of chemicals; } \\
\text { reduction of waste }\end{array}$ \\
\hline Catalyst selectivity & $\begin{array}{l}\text { Very strong acids cause side reactions } \\
\text { such as Wagner-Meerwein } \\
\text { rearrangements and etherification }\end{array}$ & $\begin{array}{l}\text { Fewer side reactions as a weaker acid is } \\
\text { employed }\end{array}$ & $\begin{array}{l}\text { Conservation of energy and } \\
\text { chemicals due to higher yields, } \\
\text { purities and simpler } \\
\text { purification }\end{array}$ \\
\hline Safety & $\begin{array}{l}\text { Hazard potential through } \\
\text { inflammable, volatile solvents that are } \\
\text { dangerous to health; usage of very } \\
\text { strong acids }\end{array}$ & $\begin{array}{l}\text { ILs are not volatile or inflammable; no } \\
\text { exposure of operators through gas phase; } \\
\text { less stronger acids applied; solely GHS } \\
\text { classification: GHS05 (corrosive) }\end{array}$ & $\begin{array}{l}\text { Increased safety and lowered } \\
\text { exposure of operators }\end{array}$ \\
\hline Exposition of environment & $\begin{array}{l}\text { Almost inevitable if volatile } \\
\text { compounds are included }\end{array}$ & $\begin{array}{l}\text { No exposure of the environment through the } \\
\text { gas phase; full recovery and reuse possible }\end{array}$ & $\begin{array}{l}\text { In principle closed loop } \\
\text { processing possible; reduction } \\
\text { of chemicals and waste }\end{array}$ \\
\hline
\end{tabular}


However, this demands sophisticated knowledge about the preparation, design and properties of ILs and multiphasic systems for successful realization. One should also keep in mind the competition of the novel processes with other approaches for more sustainable chemistry such as the elimination of solvent or the use of aqueous systems if feasible. Although the combined approach presented here has many benefits for a greener chemistry, one should also consider its drawbacks. The most important disadvantage of the IL utilization compared to conventional solvents is the much higher amount of energy and chemicals needed for its synthesis. However, upon repeated regeneration and reuse of the IL, this drawback can be mitigated by the savings in the esterification processes itself. It can be concluded that the use of ILs as simple solvents offers significant ecological and economic benefits only in very rare cases. This is the result of their elongated synthesis protocol, problems with toxicity and low biodegradability. On the contrary, novel innovative chemical processes such as the multiphase catalysis discussed here, are highly promising for a more sustainable chemistry. As a result of the high production cost of ILs, the implementation of ILs in closed loop processes (as realized in the BASIL ${ }^{\mathrm{TM}}$ process) is demanded. This is usually quite easy to realize by the adjustable IL properties. The take-home-message of these experiments is that for the assessment of process sustainability, the overall life cycle of all chemicals included need to be considered rather than just the final process step as unfortunately often done even from experienced researchers.

\section{Conclusions}

Ionic liquids as novel advanced liquid materials offer many benefits for a more sustainable chemistry. In this context, they were (and are) often discussed as safe, non-volatile alternatives to conventional solvents. The aim for the reduction and replacement of hazardous solvents is probably the largest field of green chemistry as the unsustainable use of VOCs has an immense negative ecological impact. Therefore, many up to date researches focus on the further understanding of this solvent class and its implementation into improved or new technologies with a higher degree of sustainability. However, the classification of ILs as overall 'green solvents' is now addressed more critically than in the early days. The concerns are about the elongated IL synthesis with often critical reagents, unsolved questions regarding toxicity, accumulation and biodegradation. These aspects make the widespread use of ILs as substitute for conventional solvents very unlikely. However, if their special properties such as multifunctional use, tunability and recyclability are exploited for novel intelligent process designs, they may help to save significant amounts of chemicals and energy. This offers more sustainable and safer processes especially if synergistic effects with other approaches for improved sustainability can be achieved. One very promising strategy is the utilization of ILs with different functions in multiphasic catalysis for easy product isolation and catalyst/solvent recovery. These strategies are similar to well-developed industrial processes such as the $\mathrm{RCH} / \mathrm{RP}$ or $\mathrm{BASIL}{ }^{\mathrm{TM}}$ process. In the experiments presented here we have shown how these concepts from chemical science and industry can be transferred into simple didactical experiments. These experiments utilize the multifunctional ionic liquid $[\mathrm{BMIM}]\left[\mathrm{HSO}_{4}\right]$ that acts as simultaneously as solvent, Brønsted acidic catalyst and dehydrating agent. In the simple experiments, several examples for esterification are presented. The course of the reaction can be monitored by the formation of an organic product phase that is immiscible with the initial homogenous ionic liquid. The products can be identified by their characteristic scent so that no complex characterization methods are necessary. After the reaction, the IL can be regenerated by removal of the volatile contaminants in vacuum, thus demonstrating the facile recyclability for closed-loop processing. This method offers many ecological and economic benefits compared to the conventional esterification that should be discussed in addition to the experiments. Nevertheless, there are also important drawbacks from the elongated, resource consuming synthesis of the IL. For the evaluation of the process sustainability, the overall steps from the production of the included chemicals to the final usage need to be considered. In the past (and sometimes also in the present), the sustainability evaluation was often focused solely on the process itself, where IL usually have clear advantages over VOCs. Therefore, intelligent process design with the use of recyclable ILs as 'beyond simple solvents' combined with other green chemistry strategies are highly demanded. An example for safer processes with reduced chemical and energetic input on the pilot scale is the cellulose processing with ILs that allows the utilization of renewable resources in addition to all the other mentioned advantages of ILs [49].

\section{Conflicts of Interest}

There are no conflicts of interest to declare.

\section{Acknowledgements}

The authors thank the Deutsche Bundesstiftung Umwelt (DBU) for financial support (Grant number Az. 31925-41; Entwicklung, Erprobung und Etablierung neuer experimenteller Umweltbildungsangebote zum Themengebiet ,Ionische Flüssigkeiten und ihre ökologische Relevanz' im Chemie-Schülerlabor).

\section{References}

[1] Rauber, D., Huwer, J., Natter, H., Hempelmann, R., Nachhaltige Chemie mit Ionischen Flüssigkeiten in Experimenten für Schule und Schülerlabor, 184 pages, LernortLabor, Berlin, 2018.

[2] MacFarlane, D.R., Tachikawa, N., Forsyth, M., Pringle, J.M., Howlett, P.C., Elliott, G.D., Davis, J.H., Watanabe, M., Simon, P., Angell, C.A., Energy applications of ionic liquids, Energy Environ. Sci., 7, 2014, 232-250

[3] Plechkova, N. V., Seddon, K.R., Ionic Liquids:"Designer" Solvents for Green Chemistry, in: Methods Reagents Green Chem. John Wiley \& Sons, Inc., Hoboken, NJ, USA, n.d.: pp. 103-130.

[4] Giernoth, R., Task-Specific Ionic Liquids, Angew. Chemie Int. Ed., 49, 2010, 2834-2839. 
[5] Jessop, P.G., Searching for green solvents, Green Chem., 13, 2011, 1391.

[6] Ranke, J., Stolte, S., Störmann, R., Arning, J., Jastorff, B., Design of Sustainable Chemical Products - The Example of Ionic Liquids, Chem. Rev., 107, 2007, 2183-2206.

[7] Pellowska, M., Handel, R., Bader, H.J., Flint, A., Ionische Flüssigkeiten im Schulunterricht, CHEMKON., 21, 2014, 117-122.

[8] Stark, A., Ott, D., Kralisch, D., Kreisel, G., Ondruschka, B., Ionic Liquids and Green Chemistry: A Lab Experiment, J. Chem. Educ., 87, 2010, 196-201.

[9] United Nations General Assembly, Transforming our world: the 2030 Agenda for Sustainable Development, 2015.

[10] Reichardt, C., Welton, T., Solvents and Solvent Effects in Organic Chemistry, Wiley-VCH Verlag GmbH \& Co. KGaA, Weinheim, Germany, 2010.

[11] Clark, J., Farmer, T., Hunt, A., Sherwood, J., Opportunities for Bio-Based Solvents Created as Petrochemical and Fuel Products Transition towards Renewable Resources, Int. J. Mol. Sci., 16, 2015, 17101-17159.

[12] Holbrey, J.D., Rogers, R.D., Green Chemistry and Ionic Liquids: Synergies and Ironies, in: Ion. Liq., 2002: pp. 2-14.

[13] Anastas, P., Eghbali, N., Green Chemistry: Principles and Practice, Chem. Soc. Rev., 39, 2010, 301-312.

[14] Clarke, C.J., Tu, W.-C., Levers, O., Bröhl, A., Hallett, J.P., Green and Sustainable Solvents in Chemical Processes, Chem. Rev., 2018, acs.chemrev.7b00571.

[15] Earle, M.J., Seddon, K.R., Ionic liquids. Green solvents for the future, Pure Appl. Chem., 72, 2000, 1391-1398.

[16] Plechkova, N. V, Seddon, K.R., Applications of ionic liquids in the chemical industry, Chem. Soc. Rev., 37, 2008, 123-150.

[17] Warner, P.A.J., Green Chemistry: Theory and Practice, Oxford University Press, 2000.

[18] Zhao, D., Liao, Y., Zhang, Z., Toxicity of Ionic Liquids, CLEANSoil, Air, Water., 35, 2007, 42-48.

[19] Marr, P.C., Marr, A.C., Ionic liquid gel materials: applications in green and sustainable chemistry, Green Chem., 18, 2016, 105-128.

[20] Liu, Q.-P., Hou, X.-D., Li, N., Zong, M.-H., Ionic liquids from renewable biomaterials: synthesis, characterization and application in the pretreatment of biomass, Green Chem., 14, 2012, 304-307.

[21] Markiewicz, M., Maszkowska, J., Nardello-Rataj, V., Stolte, S., Readily biodegradable and low-toxic biocompatible ionic liquids for cellulose processing, $R S C A d v$., 6, 2016, 87325-87331.

[22] Chen, L., Mullen, G.E., Le Roch, M., Cassity, C.G., Gouault, N., Fadamiro, H.Y., Barletta, R.E., O'Brien, R.A., Sykora, R.E., Stenson, A.C., West, K.N., Horne, H.E., Hendrich, J.M., Xiang, K.R., Davis, J.H., On the Formation of a Protic Ionic Liquid in Nature, Angew. Chemie Int. Ed., 53, 2014, 11762-11765.

[23] Jessop, P.G., Fundamental properties and practical applications of ionic liquids: concluding remarks, Faraday Discuss., 206, 2018, 587-601.

[24] Welton, T., Ionic liquids in Green Chemistry, Green Chem., 13, $2011,225$.

[25] Zhang, Y., Bakshi, B.R., Demessie, E.S., Life Cycle Assessment of an Ionic Liquid versus Molecular Solvents and Their Applications, Environ. Sci. Technol., 42, 2008, 1724-1730.

[26] Handy, S.T., Greener Solvents: Room Temperature Ionic Liquids from Biorenewable Sources, Chem. - A Eur. J., 9, 2003, 2938-2944.

[27] Varma, R.S., Namboodiri, V. V., An expeditious solvent-free route to ionic liquids using microwaves, Chem. Commun., 2001, 643-644.

[28] Jordan, A., Gathergood, N., Biodegradation of ionic liquids - a critical review, Chem. Soc. Rev., 44, 2015, 8200-8237.

[29] Thuy Pham, T.P., Cho, C.-W., Yun, Y.-S., Environmental fate and toxicity of ionic liquids: A review, Water Res., 44, 2010, 352-372.

[30] Cvjetko Bubalo, M., Radošević, K., Radojčić Redovniković, I., Halambek, J., Gaurina Srček, V., A brief overview of the potential environmental hazards of ionic liquids, Ecotoxicol. Environ. Saf., 99, 2014, 1-12.

[31] Kunz, W., Häckl, K., The hype with ionic liquids as solvents, Chem. Phys. Lett., 661, 2016, 6-12.

[32] Zhang, J., Wu, J., Yu, J., Zhang, X., He, J., Zhang, J., Application of ionic liquids for dissolving cellulose and fabricating cellulosebased materials: state of the art and future trends, Mater. Chem. Front., 1, 2017, 1273-1290.

[33] Zhu, X., Peng, C., Chen, H., Chen, Q., Zhao, Z.K., Zheng, Q., Xie, H., Opportunities of Ionic Liquids for Lignin Utilization from Biorefinery, ChemistrySelect., 3, 2018, 7945-7962.

[34] Rauber, D., Conrad, M., Huwer, J., Natter, H., Hempelmann, R., Demonstrating Sustainable Biomass Utilization and Processing Using Ionic Liquids - An Introduction to Undergraduate Chemistry Laboratories, World J. Chem. Educ., 5, 2017, 158-163.

[35] Kohlpaintner, C.W., Fischer, R.W., Cornils, B., Aqueous biphasic catalysis: Ruhrchemie/Rhône-Poulenc oxo process, Appl. Catal. A Gen., 221, 2001, 219-225.

[36] Hibbel, J., Wiebus, E., Cornils, B., 75 Jahre Hydroformylierung Oxoreaktoren und Oxoanlagen der Ruhrchemie $\mathrm{AG}$ und der Oxea GmbH von 1938 bis 2013, Chemie Ing. Tech., 85, 2013, 1853-1871.

[37] Maase, M., Massonne, K., Vagt, U., BASIL ${ }^{\mathrm{TM}}$-BASF's Processes Based on Ionic Liquids, Chem Files., 2002, 4.

[38] Gutowski, K.E., Industrial uses and applications of ionic liquids, Phys. Sci. Rev., 3, 2018, 1-10.

[39] Saling, P., Maase, M., Vagt, U., Eco-efficiency Analysis of an Industrially Implemented Ionic Liquid-based Process - the BASF BASIL Process, in: Handb. Green Chem., Wiley-VCH Verlag GmbH \& Co. KGaA, Weinheim, Germany, 2010.

[40] Otera, J., Nishikido, J., Industrial Uses, in: Esterification Methods, React. Appl. Second Ed., Wiley-VCH, Weinheim, Germany, 2010 pp. 293-322.

[41] Jander, G., Jahr, K.-F., Maßanalyse - Theorie und Praxis der Titrationen mit chemischen und physikalischen Indikationen, 17. Auflag, De Gruyter, Berlin, 2009.

[42] Naydenov, D., Bart, H.-J., Ternary Liquid-Liquid Equilibria for Six Systems Containing Ethylacetate + Ethanol or Acetic Acid + an Imidazolium-Based Ionic Liquid with a Hydrogen Sulfate Anion at 313.2 K, J. Chem. Eng. Data., 52, 2007, 2375-2381.

[43] Gui, J., Cong, X., Liu, D., Zhang, X., Hu, Z., Sun, Z., Novel Brønsted acidic ionic liquid as efficient and reusable catalyst system for esterification, Catal. Commun., 5, 2004, 473-477.

[44] Fraga-Dubreuil, J., Bourahla, K., Rahmouni, M., Bazureau, J.P., Hamelin, J., Catalysed esterifications in room temperature ionic liquids with acidic counteranion as recyclable reaction media, Catal. Commun., 3, 2002, 185-190.

[45] Tao, D.-J., Wu, J., Wang, Z.-Z., Lu, Z.-H., Yang, Z., Chen, X.-S., $\mathrm{SO} 3 \mathrm{H}$-functionalized Brønsted acidic ionic liquids as efficient catalysts for the synthesis of isoamyl salicylate, $R S C A d v ., 4,2014$ $1-7$

[46] Wang, Q., Wu, Z.M., Li, Y., Tan, Y., Liu, N., Liu, Y., The efficient hydroxyalkylation of phenol with formaldehyde to bisphenol $\mathrm{F}$ over a thermoregulated phase-separable reaction system containing a water-soluble Brønsted acidic ionic liquid, RSC Adv., 4, 2014, 33466-33473.

[47] Jasiak, K., Siewniak, A., Kopczyńska, K., Chrobok, A., Baj, S., Hydrogensulphate ionic liquids as an efficient catalyst for the synthesis of cyclic carbonates from carbon dioxide and epoxides, $J$. Chem. Technol. Biotechnol., 91, 2016, 2827-2833.

[48] Gao, H., Guo, C., Xing, J., Zhao, J., Liu, H., Extraction and oxidative desulfurization of diesel fuel catalyzed by a Brønsted acidic ionic liquid at room temperature, Green Chem., 12, 2010, 1220.

[49] Mahmood, H., Moniruzzaman, M., Yusup, S., Welton, T., Ionic liquids assisted processing of renewable resources for the fabrication of biodegradable composite materials, Green Chem., 19, 2017, 2051-2075. 